\title{
A retrospective single-centre analysis of the oncological impact of LI-RADS classification applied to Metroticket 2.0 calculator in liver transplantation: every nodule matters
}

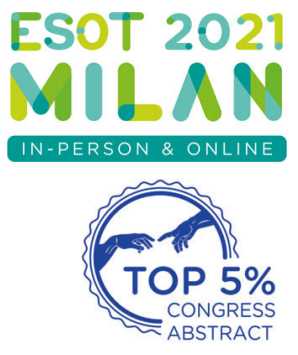

Leonardo Centonze $^{1}$ (D), Stefano Di Sandro ${ }^{1,2}$ (D, Andrea Lauterio ${ }^{1}$ (D), Riccardo De Carlis $^{1}$ (D), Cristiano Sgrazzutti ${ }^{3}$, Cristina Ciulli ${ }^{1,4}$, Ivan Vella ${ }^{1}$ (D), Ilaria Vicentin ${ }^{3}$, Niccolò Incarbone ${ }^{1,4}$ (D), Vincenzo Bagnardi ${ }^{5}$, Angelo Vanzulli ${ }^{3,6 *}$ (D) \& Luciano De Carlis ${ }^{1,4 *}$

1 Department of General Surgery and Transplantation, Niguarda Ca' Granda Hospital, Milan, Italy

2 Hepatopancreatobiliary Surgery and Liver Transplantation Unit, University of Modena and Reggio Emilia,

Modena, Italy

3 Advanced Technologies

Department, Niguarda Ca' Granda

Hospital, Milan, Italy

4 School of Medicine, University of

Milan-Bicocca, Milan, Italy

5 Department of Statistics and

Quantitative Methods, University of

Milan-Bicocca, Milan, Italy

6 Department of Oncology and

Hemato-oncology, University of

Milan, Milan, Italy

\section{Correspondence}

Leonardo Centonze, Department of General Surgery and Transplantation, Niguarda Ca' Granda Hospital, Piazza dell'Ospedale Maggiore, 3 - 20162

Milan, Italy.

Tel.: +39 3334127815;

fax: +39 0264444891;

e-mail: leonardo.centonze@

ospedaleniguarda.it

*A.V. and L.D.C. equally contributed to this work.

\section{SUMMARY}

Although the diagnostic value of Liver Imaging Reporting and Data System (LI-RADS) protocol is well recognized in clinical practice, its role in liver transplant (LT) setting is under-explored. We sought to evaluate the oncological impact of LI-RADS classification applied to Metroticket 2.0 calculator in a single-centre retrospective cohort of transplanted hepatocellular carcinoma (HCC) patients, exploring which LI-RADS subclasses need to be considered in order to grant the best Metroticket 2.0 performance. The most recent pre-LT imaging of 245 patients undergoing LT for HCC between 2005 and 2015 was retrospectively and blindly reviewed, classifying all nodules according to LI-RADS protocol. Metroticket 2.0 accuracy was subsequently tested incorporating all vital nodules identified during multidisciplinary team (MDT) meetings attended before LI-RADS reclassification of the latest pre-LT imaging, LR-5 and LR-treatment-viable (LR-TRV), LR-4/5 and LR-TR-V, and LR-3/4/5 and LR-TR-V nodules respectively. Considering their extremely low probability for harbouring HCC, LR-1 and LR-2 nodules were not considered in this analysis. Incorporation of all HCCs identified during MDT meetings attended before LI-RADS reclassification of the latest pre-LT imaging resulted in a Metroticket $2.0 \mathrm{c}$-index of 0.72, [95\% confidence interval (CI) 0.64-0.80]. Metroticket 2.0 c-index dropped to 0.60 [95\% CI: 0.48-0.72] when LI-RADS-5 and LI-RADS-TR$\mathrm{V} \quad(P=0.0089)$ or LI-RADS-5, LI-RADS-4 and LI-RADS-TR-V $(P=0.0068)$ nodules were entered in the calculator. Conversely, addition of LI-RADS-3 HCCs raised the Metroticket 2.0 c-index to 0.65 [95\% CI: $0.54-0.86]$, resulting in a not statistically significant diversion from the original performance ( 0.72 vs. $0.65 ; P=0.08)$. Exclusion of LR-3 and LR-4 nodules from Metroticket 2.0 calculator resulted in a significant drop in its accuracy. Every nodule with an intermediate-to-high probability of harbouring HCC according to LI-RADS protocol seems to contribute to tumour burden and should be entered in the Metroticket 2.0 calculator in order to grant appropriate performance. 


\section{Key words}

hepatocellular carcinoma, LI-RADS, liver imaging, liver transplantation, Metroticket 2.0, tumour staging

\section{Introduction}

Hepatocellular carcinoma (HCC) represents one of the leading causes of cancer-related death worldwide, showing increasing incidence in the last decades [1]. Liver transplantation (LT) emerged as the most effective treatment for HCC, which gradually became one of the leading indications for LT [2]. Since the release of Milan criteria in the mid-90s [3], several models [4-8] have been developed in order to expand eligibility for transplantation, optimize patient selection and ultimately predict survival; despite that, Milan criteria still represent the universally accepted benchmarks for transplant eligibility in several allocation systems in Europe [9] and the United States [10].

Every prognostic model developed so far entails the number and size of HCC nodules as major determinants of post-transplant outcomes: according to the European Association for the Study of the Liver (EASL) [11], American Association for the Study of Liver Diseases (AASLD) [12] and Organ Procurement and Transplantation Network/United Network for Organ Sharing (OPTN/UNOS) [10] guidelines, these data are obtained from contrast-enhanced imaging performed before LT.

Evolution of imaging techniques led to a refinement and standardization of HCC diagnostic criteria, summarized in Liver Imaging Reporting and Data System (LIRADS) protocol released by the American Association of Radiologists [13]. LI-RADS protocol provides HCCspecific diagnostic accuracy for each LI-RADS subclass, ranging from $38 \%$ in LR-3 up to $95 \%$ in LR-5 nodules $[13,14]$, and has been recently endorsed in AASLD guidelines for HCC management [12]; despite this widespread application, there is currently little evidence about its role in LT setting.

The main issue concerning the application of LIRADS protocol in pretransplant setting is whether to include only LR-5 nodules or to consider also other LIRADS classes with intermediate probability of harbouring HCC during preoperative workup, as predicted outcomes are deeply influenced by the number of HCC nodules that contribute to tumour burden.
The aim of this study was to evaluate the prognostic impact of LI-RADS protocol applied to Metroticket 2.0 calculator [8] in a single-centre retrospective cohort of transplanted HCC patients.

\section{Materials and methods}

Study protocol followed the 2000 Declaration of Helsinki and the 2008 Declaration of Istanbul ethical guidelines; all the subjects involved in the study gave their explicit informed consent for data collection and publication.

Local ethical committees' review of the protocol deemed that formal approval was not required owing to the retrospective, observational and anonymous nature of this study. Results are reported according to Strengthening the Reporting of Observational Studies in Epidemiology (STROBE) [15].

\section{Study design}

The study enrolled all adult patients (age $\geq 18$ years) that were listed and underwent LT for HCC between 2005 and 2015, with an available alpha-foetoprotein $(\alpha-$ FP), a retrievable contrast-enhanced imaging performed within 4 months before LT, and a proven HCC at explant pathology, with no restrictions on whether LT was the first-line treatment option or followed neoadjuvant treatments, including liver resection.

In a first step, we evaluated the Metroticket 2.0 [8] prognostic accuracy incorporating the number and size of HCC nodules that were identified during multidisciplinary team (MDT) meetings: such data were retrieved from our prospectively recorded internal database.

Notably, MDT meetings were attended before the blinded reclassification of the last pre-LT imaging according to 2018 LI-RADS protocol: in this setting, HCC diagnosis followed the noninvasive EASL criteria [11].

The most recent pre-LT contrast-enhanced studies (either computed tomography or magnetic resonance 
imaging) were retrospectively and blindly analysed by three senior radiologists with 30-, 15- and 10-year experience in liver imaging in order to classify all nodules according to 2018 LI-RADS protocol, [13] applying ancillary features when feasible. Every nodule that fitted LR-3, LR-4 and LR-5 categories was recorded, while LR-1 and LR-2 nodules were not included in this analysis because of their extremely low probability (ranging from 0 to $16 \%$ ) for harbouring HCC $[13,14]$. For those patients who underwent neoadjuvant treatments, treated nodules were classified as viable (LR-TR-V), equivocal (LR-TR-E), nonviable (LR-TR-NV) and nonevaluable (LR-TR-NE), according to LI-RADS protocol [13]. Patients for whom it was not possible to assign a LIRADS category (i.e. Patients with available but incomplete preoperative imaging - i.e. missing one or more phases of the contrast-enhanced study - or patients who did not fit the inclusion criteria for LI-RADS application) were excluded. As already stated, LI-RADS classification was applied retrospectively, so it did not affect perioperative management.

In the second step of our analysis, Metroticket 2.0 was re-calculated using the data derived from the blinded and retrospective revision of the latest pre-LT imaging according to LI-RADS protocol, including only LR-5 and LR-TR-V, LR-4/5 and LR-TR-V, and LR-3/4/ 5 and LR-TR-V nodules respectively. Since Metroticket 2.0 was built to evaluate only viable nodules [8], LRTR-NV, LR-TR-E and LR-TR-NE were excluded from this second step of the analysis.

\section{Perioperative management}

Preoperative HCC diagnosis was made according to noninvasive EASL criteria [11]. All cases were discussed during MDT meetings involving surgeons, hepatologists, radiologists and anaesthesiologists in order to assess transplant eligibility and plan bridging and downstaging.

Patient selection followed the Milan Criteria [3] until 2008 and the up-to-seven criteria from 2009 until 2015 [6]. Patients diagnosed with a tumour stage beyond selection criteria were listed for LT only after effective downstaging, whenever feasible. Extrahepatic tumour, macrovascular invasion and age $>70$ years were considered as contraindications for LT. Tumour response after downstaging or bridging was prospectively assessed on most recent pretransplant imaging using the modified Response Evaluation Criteria in Solid Tumours (mRECIST) criteria [16] since their release, and retrospectively for cases before 2010 .
LT candidates routinely underwent imaging reassessment and complete tumour restaging approximately every 3 months while on the waiting list: each case was systematically rediscussed according to the last available imaging in a MDT setting, in order to confirm transplant eligibility and assess patient prioritization. All transplanted patients underwent life-long follow-up according to EASL guidelines [11].

\section{Data collection and definition}

All data were retrieved from a single universityaffiliated, hepato-pancreato-biliary and transplant teaching centre prospective database. Several patients and tumour characteristics were considered at different time points from patient listing to transplantation, as follows: 1. At listing: patient age [years]; patient gender; aetiology of liver disease; number and size $[\mathrm{mm}]$ of HCC nodules (diagnosed accordingly to noninvasive EASL criteria [11]); $\alpha$-FP $[\mathrm{ng} / \mathrm{ml}]$; model for end-stage liver disease (MELD) score; tumour staging according to Milan-criteria [3];

2. At transplantation: donor type, defined as donation after brain death (DBD) donor, donation after cardiac death (DCD) donor or living donor liver transplantation (LDLT); time-lapse between listing and transplantation [months]; time-lapse between last radiological assessment and transplantation [months]; number of neoadjuvant treatments (including locoregional and surgical approaches with both bridging or downstaging intent); treatment response according to mRECIST criteria [16]; number and size [mm] of vital HCC nodules; nodule categorization according to LI-RADS [13]; $\alpha$-FP $[\mathrm{ng} / \mathrm{ml}]$; tumour staging according to Milan-criteria [3]; 3. At explant pathology: number and size $[\mathrm{mm}]$ of HCC nodules; pathological response (complete pathological response was defined as a complete absence of any residual vital tumour tissue in the entire specimen); tumour grading; microvascular invasion;

4. During follow-up: date of death or last censoring; date of recurrence or last censoring; cause of death.

\section{Statistical analysis}

Continuous data are reported as median and interquartile ranges (IQR). Categorical data are reported as counts and percentages.

The cumulative incidence of HCC-related deaths was estimated according to the method described by Kalbfleisch and Prentice, taking into account competing causes of death. 
The risk of 5-year HCC-related death for a patient, predicted by the Metroticket 2.0 prognostic model, was estimated using the following equation [8]:

$$
\begin{aligned}
5 \text {-year risk } & =1-\exp \{-\exp [(0.227 \\
& \times(\text { size of the largest vital tumour } \\
& + \text { number of vital nodules }) \\
& +0.817 \times\left(\log _{10} \alpha-\mathrm{FP}\right) \\
& \times 0.014]\} .
\end{aligned}
$$

Four different predictions were calculated, using in the parameter 'size of the largest vital tumour + number of vital nodules' the information derived from all the nodules identified during the MDT meetings (attended before the retrospective blinded categorization of HCC nodules according to LI-RADS protocol), or only from LR-5 and LR-TR-V, LR-4/5 and LR-TR-V, and LR-3/4/5 and LR-TR-V nodules respectively.

The discriminatory abilities of the four different models were evaluated using Wolbers c-index for regression in the presence of competing risks [17], presented with 95\% confidence intervals (CI); p-values were calculated using bootstrap methods.

Finally, to verify the results of LI-RADS application to Metroticket 2.0 model, we calculated the predicted risk of 5-year HCC-related death using Fine-Gray regression considering HCC-related death as the event of interest and $\alpha-\mathrm{FP}$, number of vital nodules and diameter of the larger vital nodule as covariates.

All reported $P$-values were two-sided, and a $P$-value of less than 0.05 was considered to indicate statistical significance. Statistical analysis was performed with SAS software, version 9.4 (SAS Institute).

\section{Results}

\section{Study population}

Flow chart for patient selection is depicted in Fig. 1.

A total of 404 patients were listed for LT from January 2005 to December 2015; 57 patients (14.4\%) dropped out for HCC progression (38 patients; 66.7\%) or other reasons (19 patients; 33.3\%) during the study period, while the remaining 347 underwent LT.

Fifty-seven patients (14.1\%) were excluded for unretrievable preoperative imaging; other 24 patients (5.9\%) were not enrolled for unavailable pretransplant $\alpha$-FP, while 13 patients $(3.2 \%)$ were excluded as their preoperative imaging was performed $>4$ months before LT; six patients $(1.5 \%)$ were not included as LI-RADS classification was not evaluable on preoperative imaging, while other 2 patients $(0.5 \%)$ did not present any HCC on explant pathology and were consequently excluded.

The final population was composed of 245 patients harbouring 567 nodules (Table 1).

\section{Patients and HCC characteristics}

Baseline characteristics of the study population are depicted in Table 2.

Study population was composed of 219 (89.4\%) men and $26(10.6 \%)$ women with a median age of 57 (IQR: 52-61) years.

At LT listing, 147 patients $(60.0 \%)$ presented a hepatitis-C viral infection, $60(24.5 \%)$ were affected by hepatitis-B and $14(5.7 \%)$ presented a hepatitis-D viral coinfection; 76 patients $(31.0 \%)$ reported alcohol abuse, while 11 patients (4.5\%) shared other aetiologies (nonalcoholic-fatty-liver-disease, haemochromatosis, cryptogenic cirrhosis etc.), with a median MELD score of 11 (IQR: 9-14) points. Focussing on HCC characteristics at listing, study population presented with a median of 1 (IQR: 1 - 2) HCC nodule, with a maximum nodule diameter of 20 (IQR: $16-30) \mathrm{mm}$ and a median $\alpha$-FP value of 9 (IQR: $5-28) \mathrm{ng} / \mathrm{ml} ; 82.7 \%$ of the population presented a Milan-in HCC. Nine (3.7\%) patients underwent liver biopsy for HCC confirmation.

Two-hundred and four patients (84.0\%) underwent a median number of 2 (IQR: 1 - 3) neoadjuvant treatments before LT, with $51(25.1 \%)$ patients treated with liver resection. According to mRECIST criteria [16], 75 (38.9\%) patients presented a complete response, 36 (18.6\%) patients showed a partial response/stable disease, while $82(42.5 \%)$ patients experienced a disease progression; $88.6 \%$ of patients resulted within Milan criteria at the time of LT.

Median waitlist time lasted 5 (IQR: 2-8) months; 206 $(84.1 \%)$ of the liver grafts were retrieved from a DBD and $3(1.2 \%)$ from a DCD donor, while $36(14.7 \%)$ patients underwent LDLT.

Thirty-six patients (17.6\%) showed a complete pathological response at explant pathology, and microvascular invasion was detected in $64(28.6 \%)$ patients.

\section{Oncological and long-term results of LT}

Median follow-up lasted 56 (IQR: 29-83) months.

Twenty-four HCC-related deaths were reported during the follow-up period, while 30 patients died from other causes, resulting in an $8.7 \%$ (95\% CI: 5.5-12.8) 


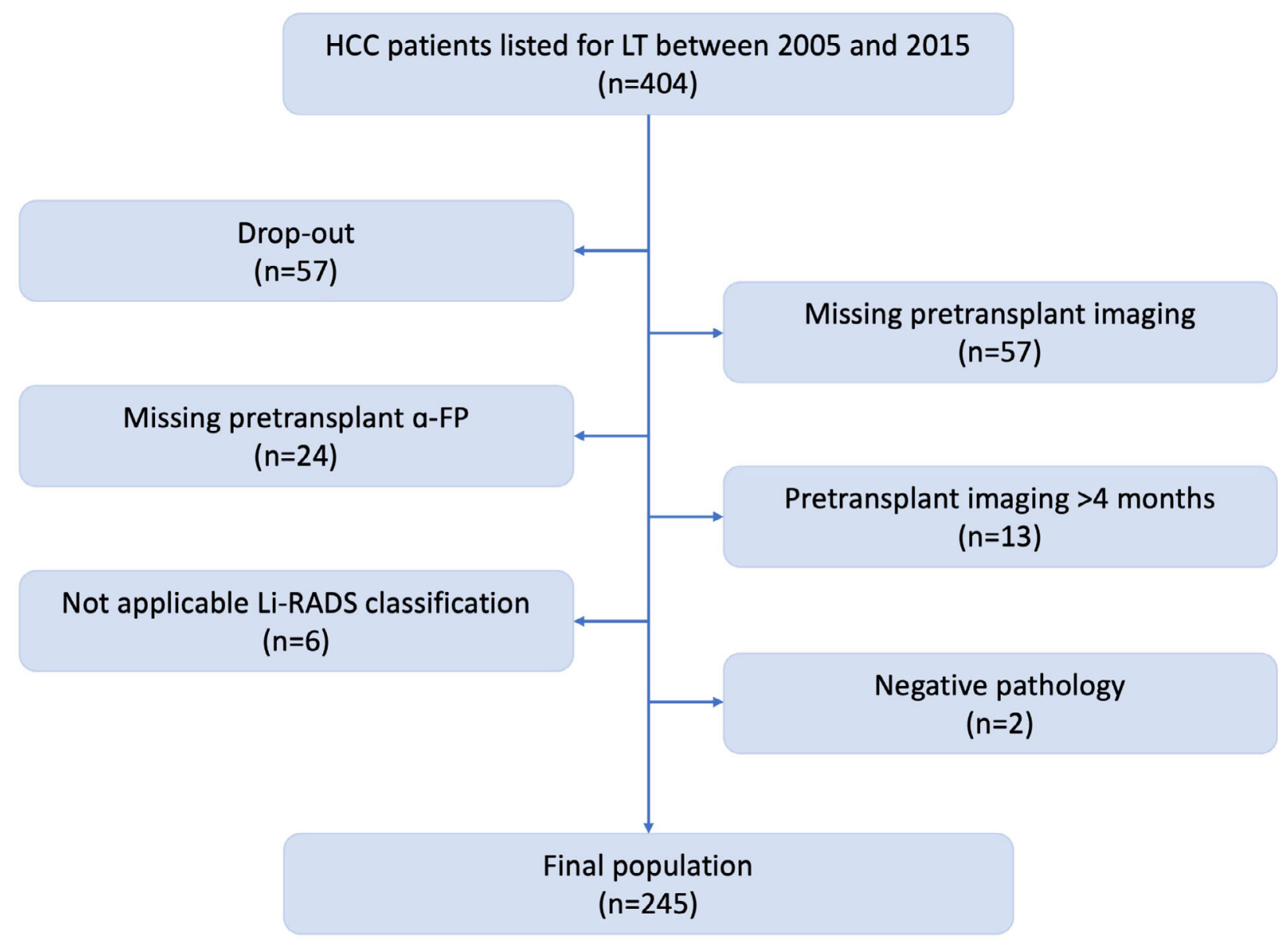

Figure 1 Flow chart for patient selection.

Table 1. Number of patients and nodules according to LI-RADS protocol

\begin{tabular}{lccccccccr}
\hline & LR-3 & LR-4 & LR-5 & LR-TR-V & LR-TR-NV & LR-TR-E & LR-NE & No nodules & Total \\
\hline Nodules & 111 & 55 & 176 & 63 & 150 & 6 & 6 & - & 567 \\
Patients* & 33 & 21 & 84 & 59 & 37 & 0 & 2 & 9 & 245 \\
\hline
\end{tabular}

LR, LI-RADS; TR-V, treatment-viable; TR-NV, treatment-nonviable; TR-E, treatment-equivocal; NE, nonevaluable.

*The highest LI-RADS class was counted.

cumulative 5-year incidence of HCC-related death (Fig. 2) and an overall 5-year survival of $80.0 \%(95 \%$ CI: 74.1-84.7).

Thirty-six HCC recurrences were observed, yielding a 5 -year cumulative incidence of recurrence of $15.7 \%$ (95\% CI: 11.1-21.0).

\section{Performance of Metroticket 2.0 calculator}

Metroticket 2.0 prognostic accuracy was first evaluated considering all the nodules identified during MDT meetings attended before the blinded preoperative imaging reclassification according to LI-RADS protocol, resulting in a c-index of 0.72 (95\% CI: $0.64-0.80)$.

Metroticket 2.0 calculator was sequentially tested including different LI-RADS subclasses and compared to the performance resulted from inclusion of all nodules regardless of LI-RADS class (Table 3): incorporation of only LR- 5 and LR-TR-V nodules resulted in a significantly lower c-index of 0.60 (95\% CI: $0.48-0.72$; $P=0.0089$ ), and the addition of LR-4 nodules yielded a similar c-index of 0.60 (95\% CI: 0.48-0.72; $P=0.0068$ ).

Conversely, inclusion of LR-3, LR-4, LR-5 and LRTR-V nodules resulted in a Metroticket $2.0 \mathrm{c}$-index of 
Table 2. Patients and HCC characteristics at listing and LT $(N=245)$.

\begin{tabular}{|c|c|}
\hline & Total $(N=245)$ \\
\hline Age (year), median (IQR) & $57(52-61)$ \\
\hline \multicolumn{2}{|l|}{ Gender, N (\%) } \\
\hline Male & $219(89.4)$ \\
\hline Female & $26(10.6)$ \\
\hline Hepatitis-C viral infection, $N(\%)$ & $147(60.0)$ \\
\hline Hepatitis-B viral infection, $N(\%)$ & $60(24.5)$ \\
\hline $\begin{array}{l}\text { Hepatitis-D viral coinfection, } N \\
(\%)\end{array}$ & $14(5.7)$ \\
\hline Alcohol abuse, $N(\%)$ & $76(31.0)$ \\
\hline Other aetiologies, $N(\%)$ & $11(4.5)$ \\
\hline Confirmation Biopsy, N (\%) & $9(3.7)$ \\
\hline $\begin{array}{l}\alpha-F P(n g / m l) \text { at listing, media } N \\
(I Q R)\end{array}$ & $9(5-28)$ \\
\hline $\begin{array}{l}\text { MELD score at listing, media } N \\
\text { (IQR) }\end{array}$ & $11(9-14)$ \\
\hline $\begin{array}{l}\text { Number of nodules at listing, } \\
\text { media } N(\mathrm{IQR})\end{array}$ & $1(1-2)$ \\
\hline $\begin{array}{l}\text { Diameter of the largest nodule } \\
(\mathrm{mm}) \text { at listing, media } N(\mathrm{IQR})\end{array}$ & $20(16-30)$ \\
\hline \multicolumn{2}{|c|}{ Within Milan criteria at listing, $N(\%)$} \\
\hline $\ln$ & $201(82.7)$ \\
\hline Out & $42(17.3)$ \\
\hline $\begin{array}{l}\text { Months from last radiological } \\
\text { assessment to } L T \text {, median (IQR) }\end{array}$ & $2(1-3)$ \\
\hline $\begin{array}{l}\text { Months from listing to } L T \text {, } \\
\text { median (IQR) }\end{array}$ & $5(2-8)$ \\
\hline \multicolumn{2}{|l|}{ Neoadjuvant treatments, $N(\%)$} \\
\hline Yes & $204(84.0)$ \\
\hline No & $39(16.0)$ \\
\hline $\begin{array}{l}\text { Number of neoadjuvant } \\
\text { treatments, median (IQR) }\end{array}$ & $2(1-3)$ \\
\hline Liver Resection, $N(\%)$ & $51(25.1)$ \\
\hline \multicolumn{2}{|c|}{$\begin{array}{l}\text { Radiological response according to mRECIST at last pre-LT } \\
\text { imaging, } N(\%)\end{array}$} \\
\hline Complete & $75(38.9)$ \\
\hline Partial/Stable & $36(18.6)$ \\
\hline Progression & $82(42.5)$ \\
\hline $\begin{array}{l}\text { Number of vital nodules at last } \\
\text { pre-LT imaging, median (IQR) }\end{array}$ & $1(0-2)$ \\
\hline $\begin{array}{l}\text { Diameter of the largest vital } \\
\text { nodule (mm) at last pre-LT } \\
\text { imaging, median (IQR) }\end{array}$ & $20(14-30)$ \\
\hline \multicolumn{2}{|c|}{ Within Milan criteria at last pre-LT imaging, $N(\%)$} \\
\hline In & $203(88.6)$ \\
\hline Out & $26(11.4)$ \\
\hline $\begin{array}{l}\text { Last } \alpha \text {-FP (ng/ml) before LT, } \\
\text { median (IQR) }\end{array}$ & $9(4-25)$ \\
\hline \multicolumn{2}{|l|}{ Donor category, $N(\%)$} \\
\hline DBD & $206(84.1)$ \\
\hline DCD & $3(1.2)$ \\
\hline LDLT & $36(14.7)$ \\
\hline \multicolumn{2}{|c|}{ Complete pathological response, $N(\%)$} \\
\hline Yes & $36(17.6)$ \\
\hline No & $209(82.4)$ \\
\hline
\end{tabular}

\section{Table 2. Continued.}

\begin{tabular}{lr}
\hline & Total $(N=245)$ \\
\hline Microvascular invasion, $N(\%)$ & \\
Yes & $64(28.6)$ \\
No & $160(71.4)$ \\
\hline
\end{tabular}

$\alpha-F P$, alpha-foetoprotein; $D B D$, donation after brain death; $D C D$, donation after cardiac death; HCC, hepatocellular carcinoma; IQR, interquartile range; $L D$, living donor; $L T$, liver transplantation; MELD, model for end-stage liver disease; mRECIST, modified Response Evaluation Criteria in Solid Tumours.

0.65 (95\% CI: 0.54-0.76), which did not significantly differ compared to the original value of 0.72 $(p=0.0800)$.

\section{Fine-Gray model prognostic accuracy}

In order to verify the results of LI-RADS application to Metroticket 2.0 model, we built a Fine-Gray Model considering HCC-related death as the event of interest and $\alpha$-FP, number of nodules and diameter of the larger nodule as covariates (Table 4).

Consideration of all nodules identified during multidisciplinary meetings attended before the blinded preoperative imaging reclassification according to LI-RADS protocol resulted in a c-index of 0.73 (95\% CI: $0.65-$ 0.81 ) incorporation of only LR-5 and LR-TR-V nodules resulted in a significantly lower c-index of $0.60(95 \%$

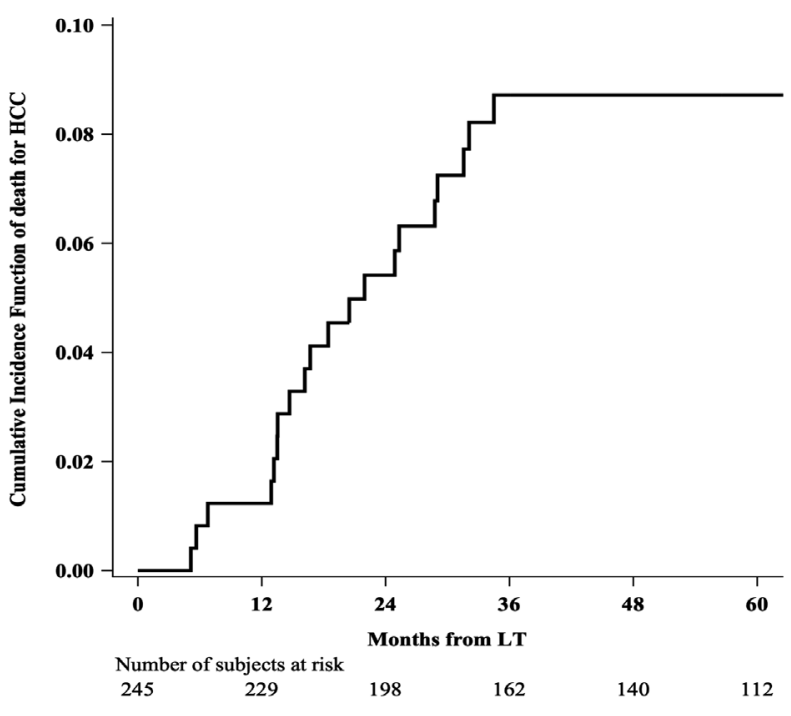

Figure 2 Cumulative incidence of death for HCC after liver transplantation $(N=245)$. 
Table 3. C-indices calculated from models using Metroticket 2.0 prediction risks, considering death for HCC as event of interest $(N=245)$.

\begin{tabular}{lccl}
\hline MetroTicket 2.0 model considering as nodules information: & C-index & $95 \% \mathrm{Cl}$ & $P$-value* \\
\hline Nodules identified during MDT meetings & 0.72 & $0.64-0.80$ & Reference \\
LR-5 and LR-TR-V nodules & 0.60 & $0.48-0.72$ & 0.009 \\
LR-4, LR-5 and LR-TR-V nodules & 0.60 & $0.48-0.72$ & 0.007 \\
LR-3, LR-4, LR-5 and LR-TR-V nodules & 0.65 & $0.54-0.76$ & 0.08 \\
\hline
\end{tabular}

MDT, multi-disciplinary team; $\mathrm{Cl}$, confidence interval.

*P-value to compare models with Li-RADS evaluation and MDT meetings derived model.

Table 4. C-indices calculated by applying Fine-Gray model considering death for HCC as event of interest and the value of $\alpha-F P$ and the sum of number of nodules and the diameter of the largest nodule as covariates $(N=245)$.

\begin{tabular}{lccl}
\hline Fine-Gray model considering as nodules information: & C-index & $95 \% \mathrm{Cl}$ & $P$-value* \\
\hline Nodules identified during MDT meetings & 0.73 & $0.65-0.81$ & Reference \\
LR-5 and LR-TR-V nodules & 0.60 & $0.48-0.72$ & 0.016 \\
LR-4, LR-5 and LR-TR-V nodules & 0.60 & $0.48-0.72$ & 0.019 \\
LR-3, LR-4, LR-5 and LR-TR-V nodules & 0.69 & $0.58-0.80$ & 0.26 \\
\hline
\end{tabular}

MDT: multi-disciplinary team; $\mathrm{Cl}$ : confidence interval.

*P-value to compare models with Li-RADS evaluation and MDT meetings derived model.

CI: $0.48-0.72 ; P=0.0160)$, while addition of LR-4 nodules yielded a similar c-index of 0.60 (95\% CI: $0.48-$ $0.72 ; P=0.0180)$.

Notably, inclusion of LR-3, LR-4, LR-5 and LR-TR-V nodules resulted in a c-index of 0.69 (95\% CI: $0.58-$ 0.80 ), which did not significantly differ compared to the original value of $0.73(P=0.2600)$.

\section{Discussion}

The Metroticket 2.0 calculator is the only prognostic index developed under a competing-risk framework and allows to estimate the HCC-specific survival starting from radiological staging and $\alpha$-FP value. [8] Such model was built on 1018 patients undergoing LT for HCC in three tertiary Italian transplant centres including nodules that fitted the EASL diagnostic criteria [11] and validated on a 341 eastern cohort from Shanghai, yielding a 5-year HCC-specific survival c-index of 0.72 .

Preoperative workup of LT candidates mandatorily includes contrast-enhanced imaging for HCC diagnosis and staging. According to the current EASL guidelines, [11] a nodule $>1 \mathrm{~cm}$ arising in a cirrhotic liver and presenting typical radiological hallmarks is eventually diagnosed as HCC. On the other hand, the OPTN/UNOS protocol [10] endorses stricter criteria for HCC

diagnosis in LT setting, with the aim to increase its specificity and contrast the allocation of organs to falsepositive patients [18].

Since its initial release in 2011, the LI-RADS protocol has been constantly refined in order to provide a standardized terminology, technique and interpretation of liver imaging. LI-RADS classification identifies different nodule subclasses with increasing probability of harbouring HCC, ranging from $38 \%$ in LR-3 up to $95 \%$ in LR-5 nodules $[13,14]$. The major difference between the EASL or OPTN/UNOS systems and LI-RADS protocol is represented by their binary classification versus the probabilistic algorithmic approach of LI-RADS [19]. The recent inclusion of LI-RADS protocol in the AASLD guidelines for HCC management [12] represented a first step towards the standardization of diagnostic approach to liver tumours, though its role in preoperative workup of LT candidate is little explored. In particular, application of LI-RADS protocol in LT setting raises the question on whether to include or exclude those observations with an intermediate probability of harbouring HCC (such as LR-3 and LR-4 nodules) from preoperative tumour staging.

As already stated, the Metroticket 2.0 identifies a continuum of outcome probabilities using morphological and biological HCC characteristics: such model 
represents a reliable tool for preoperative risk stratification of HCC transplant candidates, but could also be applied for retrospectively assess the influence of LIRADS classification on post-transplant outcomes.

Following this design, Metroticket 2.0 performance was originally tested including all the vital nodules identified during MDT meetings attended before a blinded and retrospective reclassification of pre-LT imaging according to LI-RADS protocol, yielding a $0.72 \mathrm{c}$-index; such prognostic accuracy was comparable to that accomplished in the original work that defined the calculator [8].

Our analysis showed that the exclusion of LR-3 and LR-4 nodules (which share an intermediate probability for harbouring HCC) from the Metroticket 2.0 calculator resulted in a significant drop in its prognostic accuracy, with a c-index reduction from 0.72 to 0.60 . On the other hand, inclusion of all vital nodules ranging from LR-3 to LR-5 classes raised its c-index to 0.65 , with no statistically significant difference from its original performance. Such implications were also confirmed through a Fine-Gray regression model that incorporated the same risk factors of Metroticket 2.0, with similar outcomes.

Our results could possibly rely on misinterpretation of tumour burden caused by inclusion or exclusion of different nodules according to their LI-RADS class. Since liver transplant candidates for HCC often present multinodular disease, the categorization of each nodule deeply influences preoperative staging and, consequently, the prediction power of any prognostic model.

Considering the high specificity of LR- 5 class according to LI-RADS protocol, the exclusive incorporation of this category in preoperative staging would grant the stricter patient selection, at the cost of decreased sensibility in HCC diagnosis for those nodules with lower LI-RADS classes. A similar drawback has also been highlighted in a retrospective evaluation of the OPTN/ UNOS classification system, which shares a similar high-specificity approach [18]. The reduced performance of Metroticket 2.0 calculator after exclusive consideration of LR-5 (and LR-TR-V) subclass could therefore be explained by an underestimation of tumour load because of the exclusion of other nodules with intermediate probability for harbouring HCC: although this percentage lies between 38\% for LR-3 and 74\% for LR-4 subclass [20], the presence of HCC focus eventually results in an biased estimation of the preoperative tumour burden. In this setting, the Metroticket 2.0 would erroneously predict a better outcome justifying the lower c-index of our retrospective evaluation based on actuarial HCC-specific survival.
In contrast, considering the lower specificity of LR-3 and LR-4 compared to LR-5 class, incorporation of those nodules in preoperative staging could led to an overestimation of preoperative tumour burden, with similar effects on the prognostic accuracy of the Metroticket 2.0 calculator (although clinically opposite). Such consideration might partially explain the slight nonstatistically significant difference between the Metroticket 2.0 c-indices derived from inclusion of LR-3/5 vs. all nodules identified during MDT meetings; taking into account the extremely low probability (ranging from 0 to $16 \%$ ) of LR-1 and LR-2 nodules for harbouring HCC, this nonstatistically significant difference in Metroticket 2.0 performance could also rely on a partial (although unlikely) underestimation of tumour burden because of their exclusion from the analysis.

The main limitations of this study are represented by its retrospective nature, which may imply selection and indication biases, as well as the lack of a correlation analysis of radiological and pathological characteristics for every nodule. Although the evaluation of postoperative pathology would have reinforced our conclusions (i.e. highlighting unfavourable pathological HCC characteristics [21]), it must be pointed out that pathological diagnosis is rarely performed preoperatively in common clinical practice, as documented by the 3.7\% rate of confirmatory biopsy reported in our study, while pure radiological evaluation of tumour burden represents the actual tool for preoperative staging.

A recent multicentric analysis by Cucchetti et al. [22] highlighted how the evaluation of tumour response to neoadjuvant treatments through mRECIST criteria could improve the Metroticket 2.0 prognostic accuracy by identifying HCC patients with pre-LT disease progression sharing a higher risk of tumour-related death. Despite this growing evidence supporting the pivotal role of response to neoadjuvant treatments in determining post-transplant outcomes and guide patient prioritization and liver allocation [23], this biological surrogate of tumour behaviour was not included in the Metroticket 2.0 model and consequently in this analysis: in fact, our study aim was to assess which nodules according to LI-RADS classification need to be considered in the Metroticket 2.0 in order to achieve its best accuracy rather than improve its performance. Combined evaluation of LI-RADS classification, radiological response to neoadjuvant treatments and prospective pathological analysis of the liver explants represents an interesting field for future research that could further improve HCC patient selection in LT setting.

Despite these limitations, our study supports a cautious application of LI-RADS classification in LT 
setting, suggesting the inclusion of every vital nodule with an intermediate-to-high probability of harbouring HCC according to LI-RADS protocol in order to grant the best performance of the Metroticket 2.0 model.

The results of this single-centre evaluation should be hopefully validated in larger multicentric populations in order to draw stronger conclusions.

\section{Authorship}

SDS, AV, and LC: conceptualized the study. SDS, AV, LC and VB: designed the methodology. VB: involved in formal analysis. LC, RDC, CS, CC, IV, IV and NI: involved in data curation. LC: prepared the original draft. LC, SDS, AL and RDC: reviewed and edited the manuscript. SDS, VB, AV and LDC: supervised the study. All authors have read and agreed to the published version of the manuscript.

\section{Funding}

The authors have declared no funding.

\section{Conflict of interest}

The authors have declared no conflicts of interest.

\section{Acknowledgments}

The Authors would like to thank Dr Mattia Pecorilla, Dr Alessandra Coppola and Dr Giulia Platania for their contribution in data collection and management.

\section{Data availability statement}

Data will be available upon request to the corresponding author.

\section{REFERENCES}

1. Global Burden of Disease Liver Cancer Collaboration. Akinyemiju T, Abera S, et al. The burden of primary liver cancer and underlying etiologies from 1990 to 2015 at the global, regional, and national level: results from the global burden of disease study 2015. JAMA Oncol 2017; 3: 1683.

2. Kim WR, Lake JR, Smith JM, et al. OPTN/SRTR 2016 annual data report: liver. Am J Transplant 2018; 18(Suppl 1): 172 .

3. Mazzaferro V, Regalia E, Doci R, et al. Liver transplantation for the treatment of small hepatocellular carcinomas in patients with cirrhosis. $N$ Engl $\mathrm{J}$ Med 1996; 334: 693.

4. Duffy JP, Vardanian A, Benjamin E, et al. Liver transplantation criteria for hepatocellular carcinoma should be expanded: a 22-year experience with 467 patients at UCLA. Ann Surg 2007; 246: 502; discussion 509-511.

5. Ito T, Takada Y, Ueda M, et al. Expansion of selection criteria for patients with hepatocellular carcinoma in living donor liver transplantation. Liver Transpl 2007; 13: 1637.

6. Mazzaferro V, Llovet JM, Miceli R, et al. Predicting survival after liver transplantation in patients with hepatocellular carcinoma beyond the Milan criteria: a retrospective, exploratory analysis. Lancet Oncol 2009; 10: 35.

7. Duvoux C, Roudot-Thoraval F, Decaens $\mathrm{T}$, et al. Liver transplantation for hepatocellular carcinoma: a model including $\alpha$-fetoprotein improves the performance of Milan criteria. Gastroenterology 2012; 143: 986; quiz e14e15.

8. Mazzaferro V, Sposito C, Zhou J, et al. Metroticket 2.0 model for analysis of competing risks of death after liver transplantation for hepatocellular carcinoma. Gastroenterology 2018; 154: 128.

9. De Carlis L, Di Sandro S, Centonze L, et al. Liver-allocation policies for patients affected by HCC in Europe. Curr Transplant Rep. 2016; 3: 313.

10. Wald C, Russo MW, Heimbach JK, Hussain HK, Pomfret EA, Bruix J. New OPTN/UNOS policy for liver transplant allocation: standardization of liver imaging, diagnosis, classification, and reporting of hepatocellular carcinoma. Radiology 2013; 266: 376.

11. European Association for the Study of the Liver. European Association for the Study of the Liver. EASL Clinical Practice Guidelines: Management of hepatocellular carcinoma. J Hepatol. 2018; 69: 182.

12. Marrero JA, Kulik LM, Sirlin CB, et al. Diagnosis, staging, and management of hepatocellular carcinoma: 2018 practice guidance by the American Association for the Study of Liver Diseases. Hepatology 2018; 68: 723-750. http:// dx.doi.org/10.1002/hep.29913.

13. Chernyak V, Fowler KJ, Kamaya A, et al. Liver imaging reporting and data system (LI-RADS) version 2018: imaging of hepatocellular carcinoma in at-risk patients. Radiology 2018; 289: 816.

14. Tang ES-T, Hall G, Yu D, Menard A, Hopman W, Nanji S. Predictors and cumulative frequency of hepatocellular carcinoma in high and intermediate LI-RADS lesions: a cohort study from a Canadian academic institution. Ann Surg Oncol 2019; 26: 2560.

15. von Elm E, Altman DG, Egger $M$, et al. Strengthening the reporting of observational studies in epidemiology (STROBE) statement: guidelines for reporting observational studies. $B M J$ 2007; 335806.

16. Lencioni R, Llovet JM. Modified RECIST (mRECIST) assessment for hepatocellular carcinoma. Semin Liver Dis 2010; 30: 52.

17. Wolbers M, Blanche $\mathrm{P}$, Koller MT, Witteman JCM, Gerds TA. Concordance for prognostic models with competing risks. Biostatistics. 2014; 15: 526.

18. Fowler KJ, Karimova EJ, Arauz AR, et al. Validation of organ procurement and transplant network (OPTN)/united network for organ sharing (UNOS) criteria for imaging diagnosis of hepatocellular carcinoma. Transplantation 2013; 95: 1506

19. Cunha GM, Tamayo-Murillo DE, Fowler KJ. LI-RADS and transplantation: challenges and controversies. Abdom Radiol 2021; 46: 29.

20. van der Pol CB, van der Pol CB, Lim $\mathrm{CS}$, et al. Accuracy of the liver imaging reporting and data system in computed 
tomography and magnetic resonance image analysis of hepatocellular carcinoma or overall malignancy-a systematic review. Gastroenterology 2019; 156: 976.

21. Centonze L, Di Sandro S, Lauterio A, et al. Surgical resection vs. percutaneous ablation for single hepatocellular carcinoma: exploring the impact of Li-RADS classification on oncological outcomes. Cancers. 2021; 13: 1671.

22. Cucchetti A, Serenari M, Sposito C, et al. Including mRECIST in the Metroticket 2.0 criteria improves prediction of hepatocellular carcinoma- related death after liver transplant. $J$ Hepatol 2020; 73: 342.

23. Di Sandro S, Bagnardi V, Cucchetti A, et al. From a philosophical framework to a valid prognostic staging system of the new "comprehensive assessment" for transplantable hepatocellular carcinoma. Cancers 2019; 11: 741. 